\title{
Charge-neutral nonlocal response in superconductor-InAs nanowire hybrid devices
}

\author{
A.O. Denisov, ${ }^{1,2}$ A.V. Bubis,${ }^{3,1}$ S.U. Piatrusha,${ }^{1}$ N.A. Titova, ${ }^{4}$ A.G. Nasibulin,${ }^{3,5}$ \\ J. Becker, ${ }^{6}$ J. Treu, ${ }^{6}$ D. Ruhstorfer, ${ }^{6}$ G. Koblmüller, ${ }^{6}$ E.S. Tikhonov, ${ }^{1}$ and V.S. Khrapai ${ }^{1}$ \\ ${ }^{1}$ Institute of Solid State Physics, Russian Academy of Sciences, 142432 Chernogolovka, Russian Federation \\ ${ }^{2}$ Department of Physics, Princeton University, Princeton, New Jersey 08544, USA \\ ${ }^{3}$ Skolkovo Institute of Science and Technology, Nobel street 3, 121205 Moscow, Russian Federation \\ ${ }^{4}$ Moscow Pedagogical State University, 29 Malaya Pirogovskaya St, Moscow, 119435, Russia \\ ${ }^{5}$ Aalto University, P. O. Box 16100, 00076 Aalto, Finland \\ ${ }^{6}$ Walter Schottky Institut, Physik Department, and Center for Nanotechnology and Nanomaterials, \\ Technische Universität München, Am Coulombwall 4, Garching 85748, Germany
}

\begin{abstract}
Nonlocal quasiparticle transport in normal-superconductor-normal (NSN) hybrid structures probes sub-gap states in the proximity region and is especially attractive in the context of Majorana research. Conductance measurement provides only partial information about nonlocal response composed from both electron-like and hole-like quasiparticle excitations. In this work, we show how a nonlocal shot noise measurement delivers a missing puzzle piece in NSN InAs nanowirebased devices. We demonstrate that in a trivial superconducting phase quasiparticle response is practically charge-neutral, dominated by the heat transport component with a thermal conductance being on the order of conductance quantum. This is qualitatively explained by numerous Andreev reflections of a diffusing quasiparticle, that makes its charge completely uncertain. Consistently, strong fluctuations and sign reversal are observed in the sub-gap nonlocal conductance, including occasional Andreev rectification signals. Our results prove conductance and noise as complementary measurements to characterize quasiparticle transport in superconducting proximity devices.
\end{abstract}

Nonlocal conductance measurements [1] in semiconductor-superconductor proximity structures gain renewed interest in the context of Majorana research [2-8]. The key underlying idea is that the nonlocal signals can probe global sub-gap states characteristic of a true topological phase transition [9-11]. This is in contrast to a standard two-terminal conductance [12-14] sensitive to the states near the point where the current inflows in the proximity region. Recent experiments in three-terminal NSN nanowire-based (NW-based) hybrid devices confirm conceptual power of the nonlocal conductance approach $[15,16]$.

Conductance measurement provides only partial information about quasiparticle non-equilibrium in proximity structures. A sub-gap quasiparticle entering the proximity region carries the electric charge, $q<0$ for electronlike and $q>0$ for hole-like quasiparticles, and the excitation energy $\varepsilon=|E|>0$, where $E$ is the kinetic energy relative to the chemical potential of the superconductor. On its way, apart from possible normal scattering, a quasiparticle experiences a few Andreev reflections (ARs) from the superconducting lead $[17,18]$, each time inverting the $q$ but preserving the $\varepsilon$. Thereby the AR mediates a coupling of the charge and heat (energy) transport components that is unique to proximity structures and does not occur in bulk superconductors $[19,20]$. Thus, a full characterization of the non-equilibrium can be achieved by measurement of both the electric and heat nonlocal conductances.

In this article, we investigate the nonlocal response in NSN InAs NW-based devices. We show that a quasiparticle non-equilibrium can be understood if the nonlocal conductance is accompanied by a shot noise measurement substituting the heat conductance measurement, that allows to separate the contributions of transmission processes involving even and odd number of the ARs. Experiments performed in a trivial superconducting phase demonstrate that quasiparticle transport is practically charge-neutral, so that the heat transport component dominates the nonlocal response in our devices. Our results prove shot noise as a valuable, complementary to conductance, tool to probe the sub-gap states in proximity structures.

We start the discussion from the energy diagram of the NSN NW-based hybrid structure in a nonlocal experiment sketched in Fig. 1a. Consider the case of zero temperature $T=0$ and negative bias voltage $V_{1}<0$ applied to the left normal terminal $\mathrm{N}_{1}$. The superconductor and the right normal terminal $\mathrm{N}_{2}$ are grounded, position of their chemical potential shown by the dashed line. Transmitted quasiparticles are distinguished by their energy relative to this chemical potential, $\varepsilon>0$ for electrons and $-\varepsilon$ for holes. Inside the NW quasiparticles experience ARs from the S-lead and elastic normal scattering from disorder and possibly from the $\mathrm{S} / \mathrm{NW}$ interface, inelastic scattering is absent [21]. The charge current $I_{2}$ and the heat current $J_{2}$ in the right lead read $[22,23]$ :

$$
I_{2}=-\frac{e^{2}}{h} V_{1} \Sigma \mathcal{T}_{-} ; \quad J_{2}=\frac{e^{2}}{2 h} V_{1}^{2} \Sigma \mathcal{T}_{+} ; \mathcal{T}_{ \pm} \equiv T_{21} \pm A_{21}
$$

where the positive direction for the electric current is from the lead into the scattering region and opposite for the heat current. $T_{21}$ and $A_{21}$ are the probabilities of transmission, respectively, preserving and changing a quasiparticle type, sometimes also called normal and crossed-Andreev transmission [6] and the sum over the eigenchannels is performed. Generally, the two transmission processes involve both the normal and Andreev scat- 

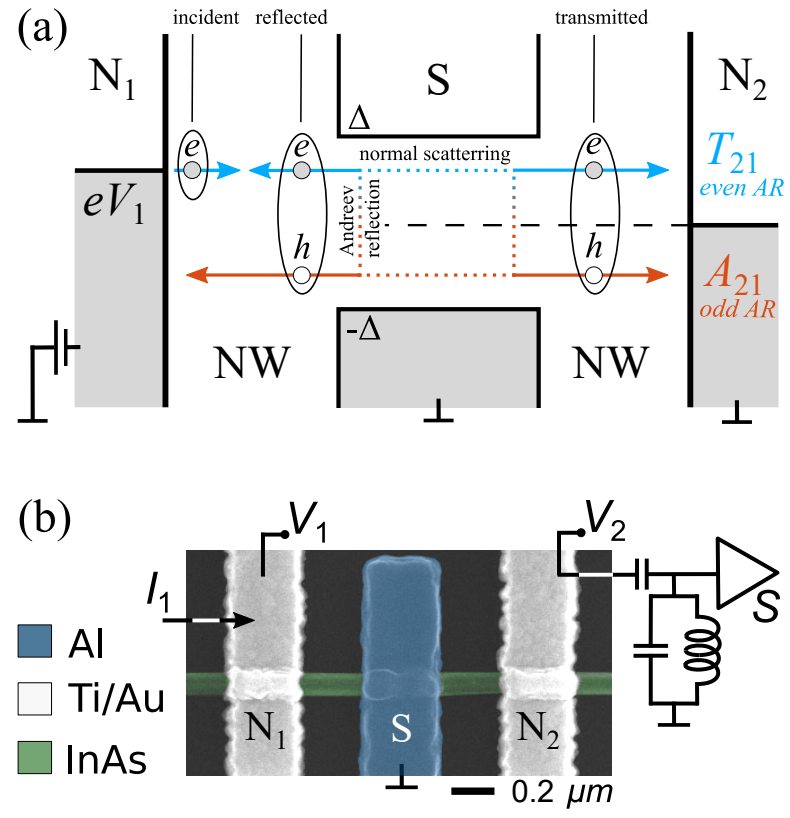

FIG. 1. (a) Energy diagram of the NSN NW-based device. In this illustration, the left normal terminal $\mathrm{N}_{1}$ is biased with voltage $V_{1}<0$, while the central S-terminal and the right $\mathrm{N}_{2}$ terminal are grounded, their chemical potential shown by the dashed line. Depending on the number of Andreev reflections, the electron incident from $\mathrm{N}_{1}$ can be transmitted towards $\mathrm{N}_{2}$ as an electron $(e)$ or hole $(h)$ with probability $T_{21}$ or $A_{21}$ correspondingly. (b) Scanning electron microscope image of the NSN-II device (false color) and the shot-noise measurement scheme used in actual experiment. Voltages $V_{1}$ and $V_{2}$ that build up in response to the current bias $I_{1}$ are measured in a quasi-four-terminal configuration.

tering and are distinguished by the parity of the number of ARs involved. Processes with an even and odd number of ARs contribute, respectively, to $T_{21}$ and $A_{21}$. Eqs. (1) imply that a simultaneous measurement of the charge and heat response permits an independent characterization of $T_{21}$ and $A_{21}$. Measurement of the heat transport is not an easy task [24-27] and we choose a different path in present experiment. We perform a shot noise measurement in a nonlocal configuration [21, 28, 29], based on the findings of Ref. [11] briefly mentioned below.

The average charge $Q_{2}$ (in units of $e$ ) transmitted in one eigenchannel in an individual scattering event equals $\left\langle Q_{2}\right\rangle=\mathcal{T}_{-}$. Its fluctuation is $\left\langle\left(\delta Q_{2}\right)^{2}\right\rangle=\left\langle Q_{2}^{2}\right\rangle-\left\langle Q_{2}\right\rangle^{2}$, where $\left\langle Q_{2}^{2}\right\rangle=\mathcal{T}_{+}$. Thus, in spirit of Ref. [30], we obtain for the spectral density of the current noise in the right lead:

$$
S_{2}=\frac{2 e^{3}}{h}\left|V_{1}\right| \Sigma\left(\mathcal{T}_{+}-\mathcal{T}_{-}^{2}\right),
$$

that contains $\mathcal{T}_{+}$and can substitute $J_{2}$ in a nonlocal measurement. In the limit of suppressed AR $A_{21} \rightarrow 0$ eq. (2) reduces to a familiar result in the normal case [31]. In this case, a nonlocal Fano factor defined as $F_{\mathrm{nl}} \equiv S_{2} / 2 e\left|I_{2}\right|$ is bounded by unity $F_{\mathrm{nl}}=1-T_{21} \leq 1$. In the opposite limit of $T_{21}=A_{21}$ the shot noise and the heat current remain finite, $S_{2} \propto J_{2}$, whereas $I_{2}=0$. Here, the nonlocal quasiparticle response is charge-neutral and $F_{\mathrm{nl}} \rightarrow \infty$.

Eqs. (1-2) illustrate our main idea that nonlocal conductance $G_{21} \equiv \partial I_{2} / \partial V_{1}$ and shot noise $S_{2}$ can serve as two complementary electrical measurements required to fully characterize quasiparticle transport. We apply this paradigm to explore the nonlocal response in InAs NW-based NSN devices. The outline of the experiment is depicted in Fig. 1b. A semiconducting InAs nanowire is equipped with an $\mathrm{S}$ terminal, made of $\mathrm{Al}$, in the middle and two $\mathrm{N}$ terminals, made of $\mathrm{Ti} / \mathrm{Au}$ bilayer, on the sides. In essence, this device represents two backto-back N-NW-S junctions sharing the same S terminal. We study two similar devices NSN-I and NSN-II which have the width of $\mathrm{S}$ terminal equal to $w=200 \mathrm{~nm}$ and $w=300 \mathrm{~nm}$, respectively. Note the absence of the plunger gates typically used to define the quantum dots [32-34] or tunnel barriers [35] adjacent to the Sterminal. In addition, for all contacts in-situ Ar milling was applied before the evaporation in order to improve the semiconductor/metal interface quality. All of this enables better coupling of the sub-gap states to the normal conducting regions. As a result, the resistances of the individual N-NW-S junctions are mainly determined by disorder scattering rather than unintentional interface reflectivity. This is confirmed by smooth gate voltage characteristics and universal diffusive value of the shot noise Fano factor in the normal state, see the data of the related experiment in Ref. [36]. Throughout the experiments the $\mathrm{S}$ terminal is grounded, terminal $\mathrm{N}_{1}$ is current biased. The terminal $\mathrm{N}_{2}$ is DC floating throughout the experiment, which allows to access the differential resistances $R_{i j} \equiv \partial V_{i} / \partial I_{j}$ in a quasi-four-terminal configuration excluding the wiring contributions. The differential conductances are obtained by inverting the measured resistance matrix, see the Supplemental Material for the details. In present experiment the non-diagonal elements are much smaller than the diagonal ones, so that approximate relations $G_{i i} \approx R_{i i}^{-1}$ and $G_{i j} \approx-R_{i j}\left(R_{11} R_{22}\right)^{-1}$ hold within a few percent accuracy. Experiments are performed at bath temperatures of $T=120-150 \mathrm{mK}$ unless stated otherwise.

Back-gate voltage $\left(V_{\mathrm{g}}\right)$ dependencies of the linear response diagonal conductances are shown in Fig. 2a. $G_{i i}$ fall in the range of a few conductance quanta and exhibit a usual sublinear increase with $V_{\mathrm{g}}$ accompanied by universal mesoscopic fluctuations. Standard procedure [37] gives a field-effect mobility of $\sim 300 \mathrm{~cm}^{2} / \mathrm{Vs}$ underestimated because of the field screening by contacts. Impact of a superconducting proximity effect on $G_{i i}$ is similar for both devices and all $V_{\mathrm{g}}$ used, typical data shown in Fig. 2b. In zero magnetic field $B$ a moderate zero-bias minimum is seen surrounded by maxima at $V= \pm \Delta / e$, where $\Delta=180 \mu \mathrm{eV}$ is the $\mathrm{Al}$ superconducting gap determined independently from the critical temperature, see Supplemental Material. In a uniform mag- 
(a)

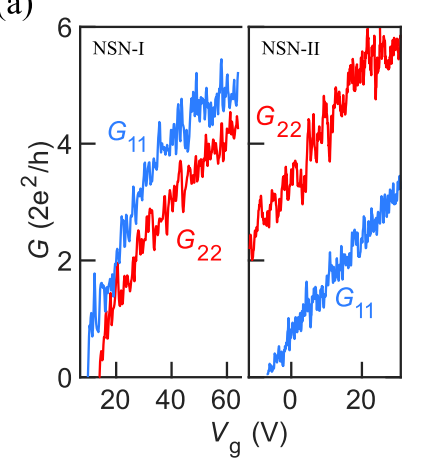

(c)

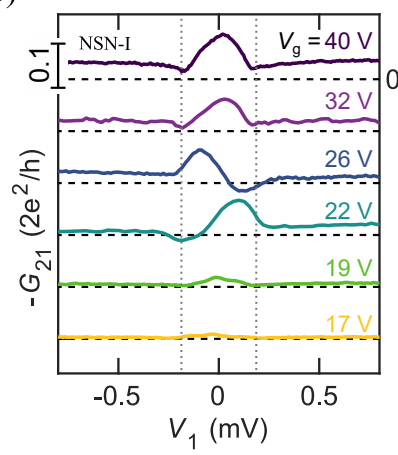

(b)

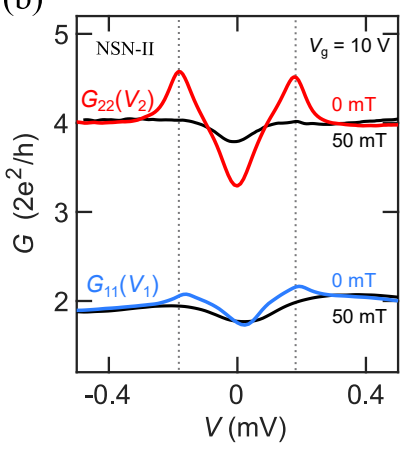

(d)

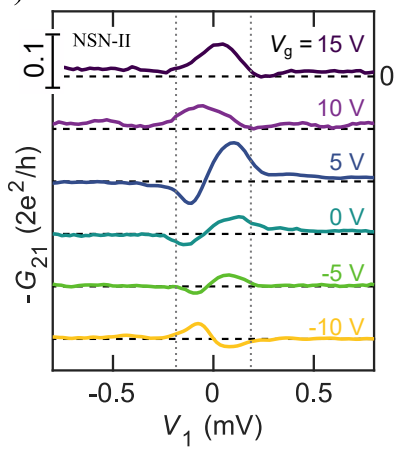

(a)

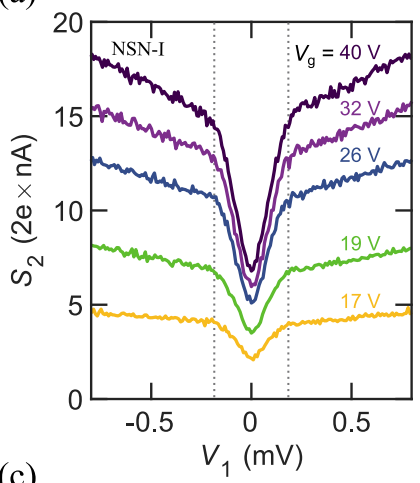

(c)

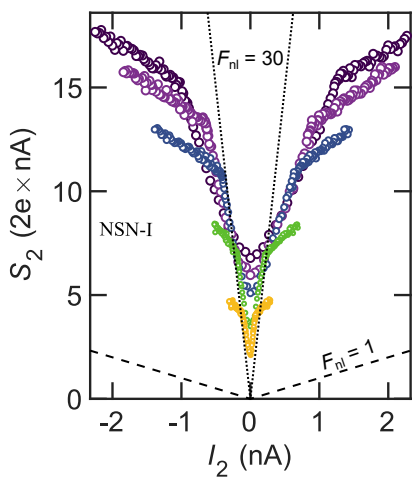

(b)
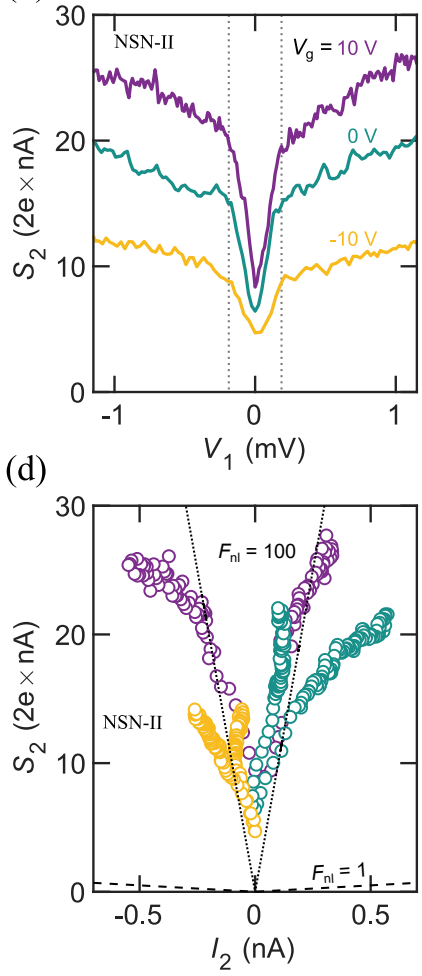

FIG. 2. (a) Zero-bias diagonal conductances versus $V_{\mathrm{g}}$ for both devices in zero magnetic field. (b) Diagonal differential conductance as a function of bias voltage in zero and high enough to suppress superconductivity magnetic fields. Dotted lines show position of the superconducting gap. (c, d) $B=$ 0 nonlocal differential conductance as a function of $V_{1}$ for several $V_{\mathrm{g}}$ values. The curves are vertically offset for clarity with zero level shown by the dashed lines. Bars indicate the ordinate scale common for all $V_{\mathrm{g}}$.

netic field of $B=50 \mathrm{mT}$ obtained with a superconducting solenoid, oriented perpendicularly to the substrate and high enough to suppress the superconductivity the minimum weakens and the maxima disappear, whereas the above-gap conductance remains unchanged. Overall, this is a standard for coherent diffusive NS junctions re-entrant behaviour $[38,39]$, with a minor effect of the interface reflectivity and/or Coulomb effects [40].

The non-diagonal conductance probes quasiparticle transport via InAs-NW section underneath the Sterminal $[4,6,7,15,16]$ and its bias dependence turns out much less universal. In Figs. 2c and $2 \mathrm{~d}$ we plot $-G_{21}$, having in mind that the negative sign corresponds to normal transmission. At sub-gap biases very different behaviour of $-G_{21}$ can be observed depending on $V_{\mathrm{g}}$, from almost symmetric with zero-bias maximum, see $V_{\mathrm{g}}=40 \mathrm{~V}$ data in device NSN-I, to strongly antisymmetric with sign inversion, see $V_{\mathrm{g}} \leq 5 \mathrm{~V}$ data in device NSN-II. By contrast, in the normal state $G_{21}$ is negative, featureless and consistent with a current transfer length of $\sim 100 \mathrm{~nm}$ determined by a residual interface reflectivity, see the Supplemental Material. Figs. 2c and 2d

FIG. 3. (a, b) Measured current noise spectral density in the right lead as a function of bias voltage on the left one. Dotted lines show positions of the superconducting gap. (c, d) Measured current noise spectral density in the right lead as a function of the nonlocal current $I_{2}$. Symbols have the same colour as the lines in panels $(\mathrm{a}, \mathrm{b})$ for the respective $V_{\mathrm{g}}$. Guide lines with Fano-factor $F_{\mathrm{nl}}=1$ and $F_{\mathrm{nl}} \gg 1$ are plotted as dashed and dotted lines correspondingly.

show that at sub-gap biases in the superconducting state $\left|G_{21}\right|$ strongly increases compared to its above-gap values, which is expected since quasiparticles are forbidden to enter the superconductor. The $G_{21}$ is much smaller than $e^{2} / h$ and occasionally changes sign, implying that $\left|\Sigma \mathcal{T}_{-}\right| \ll 1$ and fluctuates around zero as a function of energy and chemical potential. Below we present the nonlocal shot noise experiment that uncovers charge-neutral origin of the quasiparticle transport.

The layout of the shot noise measurement in a nonlocal configuration is sketched in Fig. 1b. The current fluctuations are picked up at the floating terminal $\mathrm{N}_{2}$ in response to the current between the biased terminal $\mathrm{N}_{1}$ and the grounded S-terminal. Plotted as a function of $V_{1}$ all the data in both devices feature the same qualitative behaviour shown in Figs. 3a and 3b. The shot noise spectral density $S_{2}$ starts from the Johnson-Nyquist equilibrium value $4 k_{B} T G_{22}$ at zero bias [23] and increases almost symmetrically and linear with $V_{1}$ showing a pronounced downward kink at the gap edges $\left|V_{1}\right|=\Delta / e$ marked by vertical dashed lines. Above the gap the slope drops down consistent with the fact that transmission probabil- 
ity diminishes as soon as the quasiparticles can sink in the superconductor. Nonlocal noise is more informative than the usual two-terminal noise in NS structures [33, 41-43], which exhibits only a minor reduction in the presence of sub-gap density of states [44]. According to the Eq. (2), neglecting the contributions of $\mathcal{T}_{-}^{2}$ the slope $d S_{2} / d V_{1}$ is determined by $\mathcal{T}_{+}$and allows to evaluate the linear response thermal conductance $G_{\text {th }} \equiv G_{\mathrm{th}}^{0} \Sigma \mathcal{T}_{+}$. We have $0.3<G_{\text {th }} / G_{\text {th }}^{0}<1.6$, where $G_{\text {th }}^{0}=\mathcal{L} T e^{2} / h$ is the thermal conductance quantum and $\mathcal{L}$ is the Lorenz number. This estimate of $G_{\text {th }}$ is legitimate provided $\left|\mathcal{T}_{-}\right| \ll 1$, i.e. if ballistic transmission is suppressed by disorder scattering $[18,45]$, as in our devices, or by structure geometry [46]. Note that our results manifest a drastic violation of the Wiedemann-Franz law [47] since a zero resistance of the NW region covered by the superconductor coexists with a relatively small thermal conductance via the sub-gap states.

Next we analyse the same data in terms of the nonlocal Fano factor where the nonlocal current is obtained via $I_{2}=-G_{22} V_{2}$. Here we use that the electric current caused by non-equilibrium quasiparticles is compensated by an extra current flowing in the opposite direction, so that the net current is zero in the floating configuration. This extra current flows near the Fermi level and is noiseless, since the junction $\mathrm{N}_{2}-\mathrm{NW}-\mathrm{S}$ remains essentially unbiased throughout the experiment, $\left|V_{2}\right|<k_{B} T / e$, see the Supplemental Material. Figs. $3 \mathrm{c}$ and $3 \mathrm{~d}$ plot $S_{2}$ vs $I_{2}$ for both devices. Two main features are evident. First, the symmetry inherent to $S_{2}$ vs $V_{1}$ data is in many cases lost here, since $I_{2}$ is not an anti-symmetric function of $V_{1}$. Second, the noise slope corresponds to nonlocal Fano factor values in the range $30 \lesssim F_{\mathrm{nl}} \lesssim 100$, as shown by the dotted guide lines. Such giant values of $F_{\mathrm{nl}}$ rule out a heretical interpretation that normal quasiparticle scattering from a poor quality $\mathrm{Al} / \mathrm{InAs}$ interface is the main source of nonlocal signals, that would correspond to $F_{\mathrm{nl}} \leq 1$, see the dashed guide line.

It is convenient to define the average charge of transmitted quasiparticles as the ratio between the transmitted charge and total number of transmitted quasiparticles $\left\langle q_{\mathrm{T}}\right\rangle=\Sigma \mathcal{T}_{-} / \Sigma \mathcal{T}_{+}$. The value of $\left\langle q_{\mathrm{T}}\right\rangle=1$ corresponds to the case when quasiparticles conserve their charge during the transmission process, whereas in the case $\left\langle q_{\mathrm{T}}\right\rangle=-1$ they invert the charge. As follows from the Eqs. (1-2), $\left|\left\langle q_{\mathrm{T}}\right\rangle\right|<1 / F_{\mathrm{nl}}$, i.e. the observation of a giant Fano factor implies nearly charge-neutral nonlocal quasiparticle transport with $\left|\left\langle q_{\mathrm{T}}\right\rangle\right| \ll 1$. This can be easily understood in case of a metallic diffusive NW covered by a superconductor with a transparent interface. Traversing the proximity region a quasiparticle experiences a number of ARs given on the average by $\left\langle N_{\mathrm{AR}}\right\rangle=(w / d)^{2}$, where $w$ is the width of the S-terminal and $d \approx 100 \mathrm{~nm}$ is the diameter of the NW. Given the randomness of diffusion the mean-square fluctuation is $\sqrt{\left\langle\delta N_{\mathrm{AR}}^{2}\right\rangle}=\sqrt{\left\langle N_{\mathrm{AR}}\right\rangle} \geq 2$, so that the parity of $N_{\mathrm{AR}}$ and thus the sign of the transmitted charge are completely uncertain. More rigorously, for the device NSN-II we find $\left|\left\langle q_{\mathrm{T}}\right\rangle\right|<0.01$, meaning that it (a)

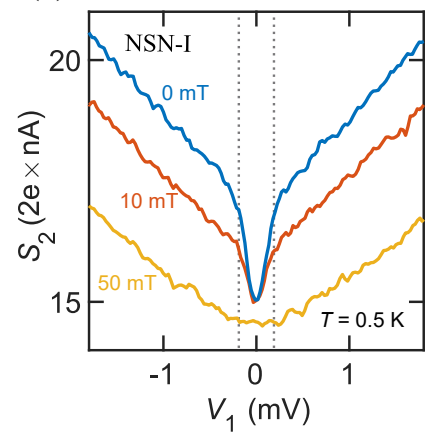

(b)

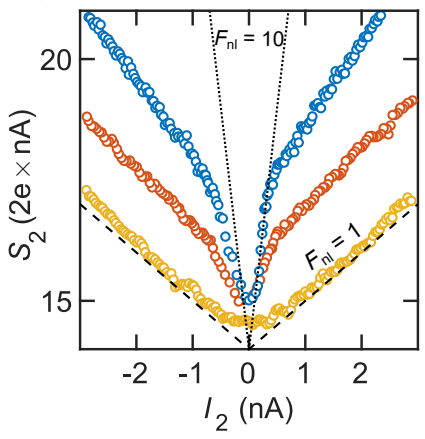

FIG. 4. (a) Evolution of the nonlocal noise spectral density $S_{2}\left(V_{1}\right)$ in magnetic field and $T=0.5 \mathrm{~K}$. Dotted lines show positions of the superconducting gap. (b) Evolution of the nonlocal noise spectral density $S_{2}\left(I_{2}\right)$ in magnetic field. Symbols have the same colour as the lines in panel (a) for the respective $B$. Guide lines with Fano-factor $F_{\mathrm{nl}}=1$ and $F_{\mathrm{nl}} \gg 1$ are plotted as dashed and dotted lines correspondingly.

takes at least a hundred quasiparticles to transmit a unit of elementary charge.

Our observations in a trivial superconducting phase have common features with the predicted nonlocal response at the topological phase transition in Majorana NWs. Here, even in presence of a moderate disorder, a finite transmission occurs in just one eigenchannel with $T_{21}=A_{21}=1 / 4$ and results in a pure heat transport characterized by a universal peak of the thermal conductance $G_{\mathrm{th}}=G_{\mathrm{th}}^{0} / 2$ [11]. While comparable in absolute value, $G_{\text {th }}$ in the present experiment demonstrates a monotonic dependence on $V_{\mathrm{g}}$. The nonlocal charge response at the topological transition restores at a finite bias, $G_{21} \propto V_{1}$, owing to the energy-dependence of the transmission probabilities, known as the Andreev rectification [4]. Similar transport features are occasionally observable in Figs. 2c and 2d, originating from mesoscopic fluctuations of $G_{21}$ around zero. This suggests that, unlike the peak in $G_{\mathrm{th}}$, Andreev rectification is not a unique signature of the topological transition, see also Ref. [16].

As a final step, we demonstrate a crossover from nearly charge-neutral to normal nonlocal quasiparticle transport in a magnetic field in the device NSN-I. Fig. 4a shows the evolution of $S_{2}$ vs $V_{1}$, taken at a bath temperature of $0.5 \mathrm{~K}$. The shot noise gradually diminishes at increasing $B$-field and the kink at the gap edge disappears concurrently with a transition of the $\mathrm{Al}$ to the normal state, see the $B=50 \mathrm{mT}$ trace. Plotted as a function of $I_{2}$ in Fig. 4b this data reveals a transition from the giant noise at sub-gap energies in the $B=0$ superconducting state to the Poissonian noise in the normal state, see the dashed guide line. In $B=0$ we find $F_{\mathrm{nl}} \sim 10$, considerably diminished compared to Fig. $3 \mathrm{c}$ as a result of thermal smearing. The Poissonian noise $F_{\mathrm{nl}} \approx 1$ in the normal state corresponds to $A_{21}=0$ and $T_{21} \ll 1$, as a result of residual interface scattering. Notably, in the 
superconducting state the slope corresponds to $F_{\mathrm{nl}}>1$ even beyond the kink, see also Figs. $3 \mathrm{c}$ and $3 \mathrm{~d}$, since the probability of the AR remains finite above the gap [19].

In summary, we performed nonlocal transport and noise experiments in InAs NW-based hybrid NSN devices. Such a combination of all-electrical measurements allows to estimate the thermal conductance and reveals a predominantly charge-neutral origin of the nonlocal response. We expect that our approach will be generally useful for the studies of non-equilibrium proximity superconductivity, including unequivocal identification of the topological phase transition in Majorana devices.

We are grateful to A.P. Higginbotham, T.M. Klapwijk, A.S. Mel'nikov and K.E. Nagaev for helpful discussions. This work was financially supported by the RSF project 19-12-00326 (fabrication, experiments in NSN-I device) and RFBR project 19-02-00898 (experiments in NSN-II device). Theoretical framework was developed under the state task of the ISSP RAS. Work at TUM was supported by the Deutsche Forschungsgemeinschaft (DFG) via project KO-4005/5-1 and Germany's Excellence Strategy-EXC-2111-390814868 (Munich Center for Quantum Science and Technology, MCQST).
[1] S. G. den Hartog, C. M. A. Kapteyn, B. J. van Wees, T. M. Klapwijk, and G. Borghs, Transport in multiterminal normal-superconductor devices: Reciprocity relations, negative and nonlocal resistances, and reentrance of the proximity effect, Phys. Rev. Lett. 77, 4954 (1996).

[2] G.-H. Lee, K.-F. Huang, D. K. Efetov, D. S. Wei, S. Hart, T. Taniguchi, K. Watanabe, A. Yacoby, and P. Kim, Inducing superconducting correlation in quantum hall edge states, Nature Physics 13, 693 (2017).

[3] M.-T. Deng, S. Vaitiekènas, E. Prada, P. San-Jose, J. Nygård, P. Krogstrup, R. Aguado, and C. M. Marcus, Nonlocality of majorana modes in hybrid nanowires, Phys. Rev. B 98, 085125 (2018).

[4] T. O. Rosdahl, A. Vuik, M. Kjaergaard, and A. R. Akhmerov, Andreev rectifier: A nonlocal conductance signature of topological phase transitions, Phys. Rev. B 97, 045421 (2018).

[5] Y.-H. Lai, J. D. Sau, and S. Das Sarma, Presence versus absence of end-to-end nonlocal conductance correlations in majorana nanowires: Majorana bound states versus andreev bound states, Phys. Rev. B 100, 045302 (2019).

[6] J. Danon, A. B. Hellenes, E. B. Hansen, L. Casparis, A. P. Higginbotham, and K. Flensberg, Nonlocal conductance spectroscopy of andreev bound states: Symmetry relations and bcs charges, Phys. Rev. Lett. 124, 036801 (2020).

[7] H. Pan, J. D. Sau, and S. Das Sarma, Three-terminal nonlocal conductance in majorana nanowires: Distinguishing topological and trivial in realistic systems with disorder and inhomogeneous potential, Phys. Rev. B 103, 014513 (2021).

[8] L. Zhao, E. G. Arnault, A. Bondarev, A. Seredinski, T. F. Q. Larson, A. W. Draelos, H. Li, K. Watanabe, T. Taniguchi, F. Amet, H. U. Baranger, and G. Finkelstein, Interference of chiral andreev edge states, Nature Physics 10.1038/s41567-020-0898-5 (2020).

[9] R. M. Lutchyn, J. D. Sau, and S. Das Sarma, Majorana fermions and a topological phase transition in semiconductor-superconductor heterostructures, Phys. Rev. Lett. 105, 077001 (2010).

[10] Y. Oreg, G. Refael, and F. von Oppen, Helical liquids and majorana bound states in quantum wires, Phys. Rev. Lett. 105, 177002 (2010).

[11] A. R. Akhmerov, J. P. Dahlhaus, F. Hassler, M. Wimmer, and C. W. J. Beenakker, Quantized conductance at the majorana phase transition in a disordered supercon- ducting wire, Phys. Rev. Lett. 106, 057001 (2011).

[12] V. Mourik, K. Zuo, S. M. Frolov, S. R. Plissard, E. P. A. M. Bakkers, and L. P. Kouwenhoven, Signatures of majorana fermions in hybrid superconductorsemiconductor nanowire devices, Science 336, 1003 (2012).

[13] A. Das, Y. Ronen, Y. Most, Y. Oreg, M. Heiblum, and H. Shtrikman, Zero-bias peaks and splitting in an al-inas nanowire topological superconductor as a signature of majorana fermions, Nature Physics 8, 887 EP (2012), article.

[14] P. Yu, J. Chen, M. Gomanko, G. Badawy, E. P. A. M. Bakkers, K. Zuo, V. Mourik, and S. M. Frolov, Nonmajorana states yield nearly quantized conductance in proximatized nanowires, Nature Physics 17, 482 (2021).

[15] G. C. Ménard, G. L. R. Anselmetti, E. A. Martinez, D. Puglia, F. K. Malinowski, J. S. Lee, S. Choi, M. Pendharkar, C. J. Palmstrøm, K. Flensberg, C. M. Marcus, L. Casparis, and A. P. Higginbotham, Conductancematrix symmetries of a three-terminal hybrid device, Phys. Rev. Lett. 124, 036802 (2020).

[16] D. Puglia, E. A. Martinez, G. C. Ménard, A. Pöschl, S. Gronin, G. C. Gardner, R. Kallaher, M. J. Manfra, C. M. Marcus, A. P. Higginbotham, and L. Casparis, Closing of the induced gap in a hybrid superconductorsemiconductor nanowire, Phys. Rev. B 103, 235201 (2021).

[17] A. F. Andreev, Thermal conductivity of the intermediate state of superconductors, JETP Letters 46, 1823 (1964).

[18] N. B. Kopnin, A. S. Mel'nikov, and V. M. Vinokur, Reentrant localization of single-particle transport in disordered andreev wires, Phys. Rev. B 70, 075310 (2004).

[19] M. Tinkham, Introduction to Superconductivity, Dover Books on Physics Series (Dover Publications, NewYork, 2004).

[20] T. T. Heikkilä, M. Silaev, P. Virtanen, and F. S. Bergeret, Thermal, electric and spin transport in superconductor/ferromagnetic-insulator structures, Progress in Surface Science 94, 100540 (2019).

[21] E. S. Tikhonov, D. V. Shovkun, D. Ercolani, F. Rossella, M. Rocci, L. Sorba, S. Roddaro, and V. S. Khrapai, Local noise in a diffusive conductor, Scientific Reports 6, 30621 EP (2016), article.

[22] N. R. Claughton and C. J. Lambert, Thermoelectric properties of mesoscopic superconductors, Phys. Rev. B 53, 6605 (1996). 
[23] M. P. Anantram and S. Datta, Current fluctuations in mesoscopic systems with andreev scattering, Phys. Rev. B 53, 16390 (1996).

[24] F. Giazotto, T. T. Heikkilä, A. Luukanen, A. M. Savin, and J. P. Pekola, Opportunities for mesoscopics in thermometry and refrigeration: Physics and applications, Rev. Mod. Phys. 78, 217 (2006).

[25] V. Chandrasekhar, Thermal transport in superconductor/normal-metal structures, Superconductor Science and Technology 22, 083001 (2009).

[26] J. T. Peltonen, P. Virtanen, M. Meschke, J. V. Koski, T. T. Heikkilä, and J. P. Pekola, Thermal conductance by the inverse proximity effect in a superconductor, Phys. Rev. Lett. 105, 097004 (2010).

[27] D. Bagrets, A. Altland, and A. Kamenev, Sinai diffusion at quasi-1d topological phase transitions, Phys. Rev. Lett. 117, 196801 (2016).

[28] E. S. Tikhonov, A. O. Denisov, S. U. Piatrusha, I. N. Khrapach, J. P. Pekola, B. Karimi, R. N. Jabdaraghi, and V. S. Khrapai, Spatial and energy resolution of electronic states by shot noise, Phys. Rev. B 102, 085417 (2020).

[29] S. Larocque, E. Pinsolle, C. Lupien, and B. Reulet, Shot noise of a temperature-biased tunnel junction, Phys. Rev. Lett. 125, 106801 (2020).

[30] R. Landauer and T. Martin, Equilibrium and shot noise in mesoscopic systems, Physica B: Condensed Matter 175, 167 (1991).

[31] Y. Blanter and M. Büttiker, Shot noise in mesoscopic conductors, Physics Reports 336, 1 (2000).

[32] L. Hofstetter, S. Csonka, J. Nygård, and C. Schönenberger, Cooper pair splitter realized in a two-quantum-dot y-junction, Nature 461, 960 (2009).

[33] A. Das, Y. Ronen, M. Heiblum, D. Mahalu, A. V. Kretinin, and H. Shtrikman, High-efficiency cooper pair splitting demonstrated by two-particle conductance resonance and positive noise cross-correlation, Nature Communications 3, 1165 EP (2012), article.

[34] M. T. Deng, S. Vaitiekenas, E. B. Hansen, J. Danon, M. Leijnse, K. Flensberg, J. Nygård, P. Krogstrup, and C. M. Marcus, Majorana bound state in a coupled quantum-dot hybrid-nanowire system, Science 354, 1557 (2016).

[35] S. M. Albrecht, A. P. Higginbotham, M. Madsen, F. Kuemmeth, T. S. Jespersen, J. Nygård, P. Krogstrup, and C. M. Marcus, Exponential protection of zero modes in majorana islands, Nature 531, 206 (2016).

[36] A. O. Denisov, A. V. Bubis, S. U. Piatrusha, N. A. Titova, A. G. Nasibulin, J. Becker, J. Treu, D. Ruhstorfer, G. Koblmueller, E. S. Tikhonov, and V. S. Khrapai, Heat-mode excitation in a proximity superconductor, arXiv:2006.09803 (2020).

[37] A. C. Ford, J. C. Ho, Y.-L. Chueh, Y.-C. Tseng, Z. Fan, J. Guo, J. Bokor, and A. Javey, Diameter-dependent electron mobility of InAs nanowires, Nano Letters 9, 360 (2009).

[38] S. G. Lachenmann, I. Friedrich, A. Förster, D. Uhlisch, and A. A. Golubov, Charge transport in superconductor/semiconductor/normal-conductor step junctions, Phys. Rev. B 56, 14108 (1997).

[39] H. Courtois, P. Charlat, P. Gandit, D. Mailly, and B. Pannetier, Journal of Low Temperature Physics 116, 187 (1999).

[40] C. W. J. Beenakker, Random-matrix theory of quantum transport, Reviews of Modern Physics 69, 731 (1997).

[41] X. Jehl, M. Sanquer, R. Calemczuk, and D. Mailly, Detection of doubled shot noise in short normal-metal/ superconductor junctions, Nature 405, 50 (2000).

[42] A. A. Kozhevnikov, R. J. Schoelkopf, and D. E. Prober, Observation of photon-assisted noise in a diffusive normal metal-superconductor junction, Phys. Rev. Lett. 84, 3398 (2000).

[43] B.-R. Choi, A. E. Hansen, T. Kontos, C. Hoffmann, S. Oberholzer, W. Belzig, C. Schönenberger, T. Akazaki, and H. Takayanagi, Shot-noise and conductance measurements of transparent superconductor/two-dimensional electron gas junctions, Phys. Rev. B 72, 024501 (2005).

[44] E. S. Tikhonov, D. V. Shovkun, M. Snelder, M. P. Stehno, Y. Huang, M. S. Golden, A. A. Golubov, A. Brinkman, and V. S. Khrapai, Andreev reflection in an $s$-type superconductor proximized 3d topological insulator, Phys. Rev. Lett. 117, 147001 (2016).

[45] A. Haim and A. Stern, Benefits of weak disorder in one-dimensional topological superconductors, Phys. Rev. Lett. 122, 126801 (2019).

[46] T. Laeven, B. Nijholt, M. Wimmer, and A. R. Akhmerov, Enhanced proximity effect in zigzag-shaped majorana josephson junctions, Phys. Rev. Lett. 125, 086802 (2020).

[47] R. Franz and G. Wiedemann, Ueber die wärmeleitungsfähigkeit der metalle, Annalen der Physik und Chemie 165, 497 (1853). 


\section{Supplemental Material}

Differential resistance and conductance matrices.

Here we outline a relation between the quantities measured in transport experiments with either fixed currents or fixed voltages applied to the normal terminals 1 and 2 in the NSN device. The voltages $V_{1}, V_{2}$ are measured with respect to the (grounded) $\mathrm{S}$ terminal. The positive direction of the currents $I_{1}, I_{2}$ is from the respective lead into the device, ensuring positive signs of the diagonal transport responses.

In a conductance experiment the independent (externally controlled) variables are voltages and the currents are measured. The elements of the differential conductance matrix are:

$$
\begin{aligned}
\left.G_{11} \equiv \frac{\partial I_{1}}{\partial V_{1}}\right|_{V_{2}=\text { const }} & \left.G_{12} \equiv \frac{\partial I_{1}}{\partial V_{2}}\right|_{V_{1}=\text { const }} \\
\left.G_{21} \equiv \frac{\partial I_{2}}{\partial V_{1}}\right|_{V_{2}=\text { const }} & \left.G_{22} \equiv \frac{\partial I_{2}}{\partial V_{2}}\right|_{V_{1}=\text { const }}
\end{aligned}
$$

A relation to the resistance experiment is straightforward. In this case externally controlled are currents and measured are voltages. As follows from the conductance matrix, the condition $I_{2}=$ const is satisfied provided $G_{21} \delta V_{1}+G_{22} \delta V_{2} \equiv \delta I_{2}=0$. With this constraint we have:

$$
\delta I_{1}=G_{11} \delta V_{1}+G_{12} \delta V_{2}=\frac{\mathrm{DET}}{G_{22}} \delta V_{1},
$$

where $\delta$ denotes an infinitesimal increment of the current/voltage and DET is the determinant of the conductance matrix DET $\equiv G_{11} G_{22}-G_{12} G_{21}$. Similar relation holds for $\delta I_{2}$ under condition of $I_{1}=$ const. Thus, for the differential resistance matrix we obtain:

$$
\begin{array}{ll}
\left.R_{11} \equiv \frac{\partial V_{1}}{\partial I_{1}}\right|_{I_{2}=\text { const }}=\frac{G_{22}}{\mathrm{DET}} & \left.R_{12} \equiv \frac{\partial V_{1}}{\partial I_{2}}\right|_{I_{1}=\text { const }}=-\frac{G_{12}}{\mathrm{DET}} \\
\left.R_{21} \equiv \frac{\partial V_{2}}{\partial I_{1}}\right|_{I_{2}=\text { const }}=-\frac{G_{21}}{\mathrm{DET}} & \left.R_{22} \equiv \frac{\partial V_{2}}{\partial I_{2}}\right|_{I_{1}=\text { const }}=\frac{G_{11}}{\mathrm{DET}}
\end{array}
$$

In present experiment the experimentally measured non-local (non-diagonal) diferential resistances are always much smaller compared to the diagonal ones. It follows that $\left|G_{12}\right|,\left|G_{21}\right| \ll\left|G_{22}\right|,\left|G_{11}\right|$, so that DET $\approx G_{11} G_{22}$. This allows to obtain the relations from the main manuscript $G_{i i} \approx R_{i i}^{-1}$ and $G_{i j} \approx-R_{i j} /\left(R_{11} R_{22}\right), i \neq j$.

\section{Nonlocal I-Vs and differential conductance.}

Measured $V_{2}\left(I_{1}\right)$ curves in the nonlocal configurations show similar non-universal behavior for both devices as evident from Fig. 2a, b. First, the nonlocal voltage signal is small $\left|V_{2}\right|<k_{\mathrm{B}} T / e \approx 13 \mu V$ and we can suppose the junction $\mathrm{N}_{2}$-NW-S to be essentially unbiased. Second, some of the I-V curves are highly non-anti-symmetric as a results we observe finite odd contribution to the non-diagonal conductance $G_{12}$ as shown in Fig. 2c. At increasing magnetic field all the sub-gap features are smeared and eventually remain featureless in sufficiently high $B=55 \mathrm{mT}$, where the superconductivity of $\mathrm{Al}$ is already suppressed.

\section{Device fabrication}

InAs nanowires grown by molecular beam epitaxy on Si substrate [S1] are ultrasonicated in isopropyl alcohol. Nanowires are drop casted on $\mathrm{Si} / \mathrm{SiO} 2(300 \mathrm{~nm})$ substrates [S2] with preliminary defined alignment marks. For superconducting contacts conventional electron beam lithography (EBL) followed by e-beam deposition of $\mathrm{Al}(150 \mathrm{~nm})$ is utilized. To obtain the ohmic contacts, in-situ Ar ion milling is performed before Al deposition in a chamber with a 
(a)

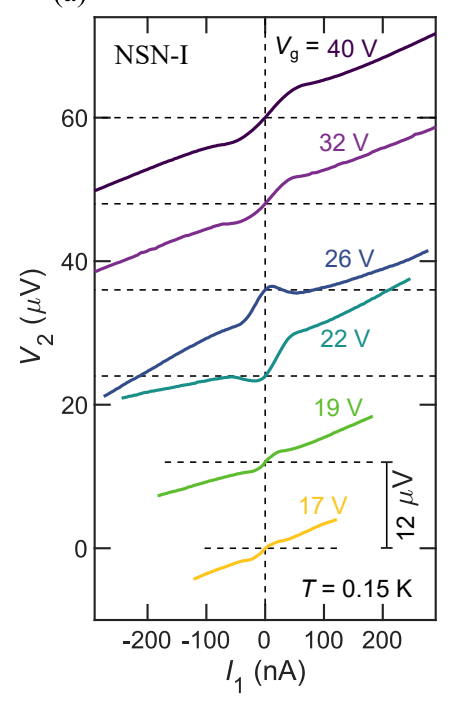

(b)

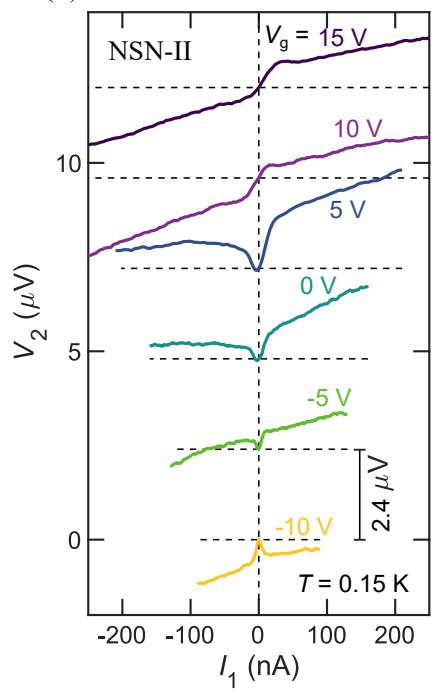

(c)

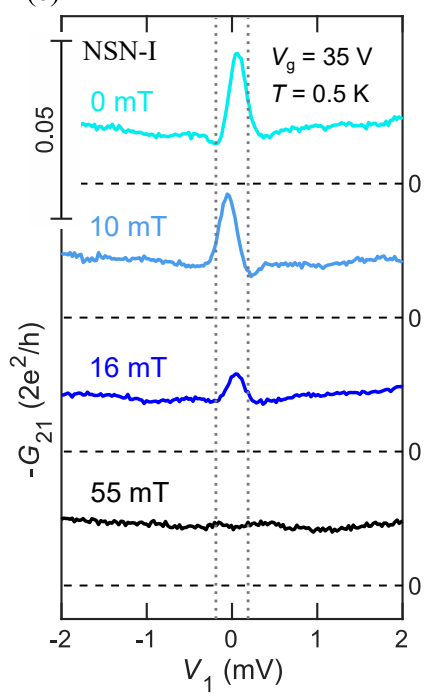

Supplemental Material Fig. 1. (a, b)Measured nonlocal I-V characteristics versus $V_{\mathrm{g}}$ for both devices in zero magnetic field. Different curves are vertically spaced with zero level shown as the dashed lines. (c) Non-diagonal conductance is plotted as a function of the bias voltage at different magnetic fields. Dotted lines show position of the superconducting gap.

base pressure below $10^{-7}$ mbar. Normal metal contacts are fabricated in two different ways (different device batches): magnetron sputtering or e-beam deposition. For sputtering (NS and NSN - I devices) in-situ Ar plasma etching is followed by sputtering of $\mathrm{Ti} / \mathrm{Au}(5 \mathrm{~nm} / 200 \mathrm{~nm})$. Normal metal contacts $\mathrm{Ti} / \mathrm{Au}(5 \mathrm{~nm} / 150 \mathrm{~nm})$ in device NSN - II are deposited in the same way as superconducting ones. 
Current transfer length estimation

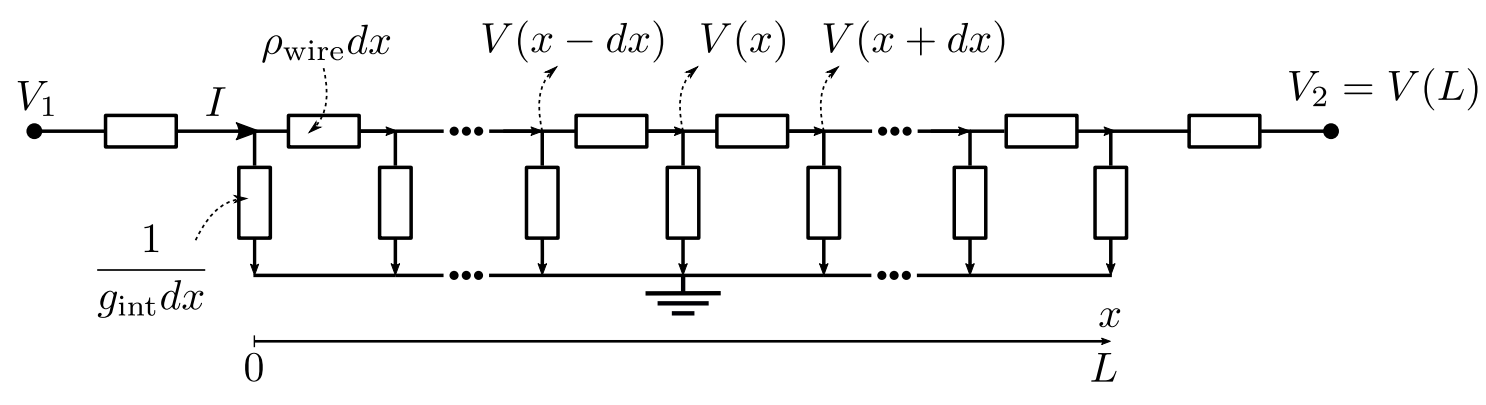

Supplemental Material Fig. 2. Effective resistance model for nanowire/superconductor interface.

To estimate the characteristic length of charge overflow within grounded $\mathrm{S}$ terminal $l_{\mathrm{T}}$ we use circuit shown in fig. 2. Here $\rho_{\text {wire }}$ and $g_{\text {int }}$ are resistance of the nanowire $(\mathrm{NW})$ and conductivity of interface per unit length respectively. In the continuous limit we can write current conservation for each point along NW/S interface:

$$
\begin{gathered}
\frac{V(x+d x)-V(x)}{\rho_{\text {wire }} d x}+\frac{V(x-d x)-V(x)}{\rho_{\text {wire }} d x}=\frac{V(x) d x}{1 / g_{\text {int }}} \\
\frac{d^{2} V(x)}{d x^{2}}=\frac{V(x)}{l_{\mathrm{T}}^{2}}, \quad l_{\mathrm{T}}=\frac{1}{\sqrt{\rho_{\text {wire }} g_{\text {int }}}}
\end{gathered}
$$

Boundary conditions including one that normal terminal N2 is floating and no current flow into it.

$$
\left.\frac{d V(x)}{d x}\right|_{x=0}=-\rho_{\text {wire }} I,\left.\frac{d V(x)}{d x}\right|_{x=L}=0
$$

Solving elementary Neumann problem we can find non-local rsistance $r_{21}$ :

$$
R_{21}=\frac{V(L)}{I}=\frac{l_{\mathrm{T}} \rho_{\text {wire }}}{\sinh \left(\frac{L}{l_{\mathrm{T}}}\right)}
$$

At high biases $\left|V_{1}\right| \gg \Delta / e$ for two measured devices (NSN-I, NSN-II) we have $R_{21} \approx 40,10 \Omega$ and $L \approx 200,300 \mathrm{~nm}$ respectively, thus $l_{\mathrm{T}} \approx 75 \mathrm{~nm}$. 


\section{Critical Temperature of Al contacts}

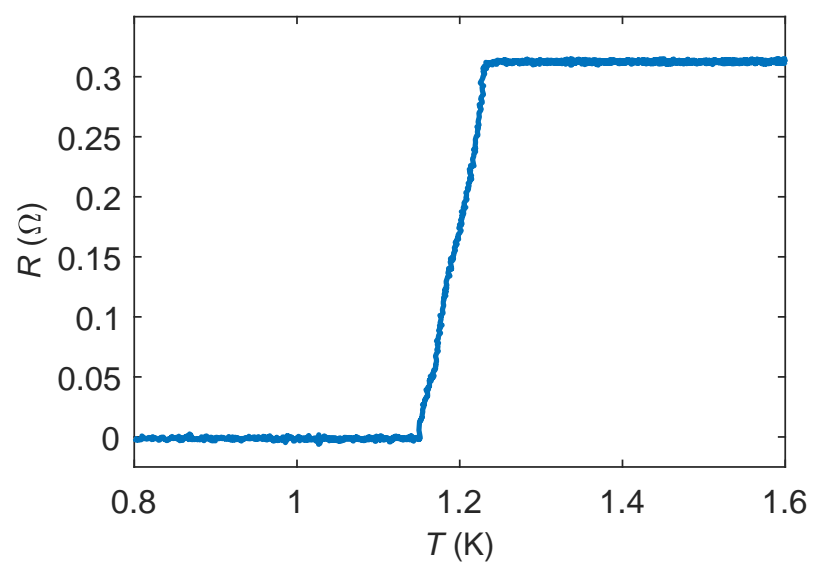

Supplemental Material Fig. 3. The resistance of a four-terminal Al strip, deposited via the same process, as the one used in the fabrication of the samples, featured in the main text.

The temperature dependence of superconducting Al, deposited via the same process as described in "Device Fabrication" was performed separately on the four-terminal Al strips, incorporated in the samples studied in [S3]. Here we present raw data (see Fig. 3), which leads to the estimate $T_{\mathrm{c}}=1.20 \pm 0.03 \mathrm{~K}$, corresponding to the superconducting energy gap of $\Delta=183 \pm 5 \mu \mathrm{V}$ in Al leads.

[S1] S. Hertenberger, D. Rudolph, M. Bichler, J. J. Finley, G. Abstreiter, and G. Koblmüller, Growth kinetics in positioncontrolled and catalyst-free InAs nanowire arrays on si(111) grown by selective area molecular beam epitaxy, Journal of Applied Physics 108, 114316 (2010).

[S2] J. Becker, S. Morkötter, J. Treu, M. Sonner, M. Speckbacher, M. Döblinger, G. Abstreiter, J. J. Finley, and G. Koblmüller, Carrier trapping and activation at short-period wurtzite/zinc-blende stacking sequences in polytypic inas nanowires, Phys. Rev. B 97, 115306 (2018).

[S3] A. V. Bubis, A. O. Denisov, S. U. Piatrusha, I. E. Batov, V. S. Khrapai, J. Becker, J. Treu, D. Ruhstorfer, and G. Koblmüller, Proximity effect and interface transparency in al/InAs-nanowire/al diffusive junctions, Semiconductor Science and Technology 32, 094007 (2017). 